\title{
ON A NEUMANN EQUILIBRIUM STATES IN ONE MODEL OF ECONOMIC DYNAMICS
}

\section{S. I. HAMIDOV}

Department of Mathematical Cybernetics

Baku State University

Baku

Azerbaijan

e-mail: sabir818@yahoo.com

\begin{abstract}
The model of economic dynamics of Neumann type is considered. With the help of the equilibrium mechanism without loss a trajectory of the model is constructed. The necessary and sufficient conditions for the existence of the equilibrium state without loss is derived.
\end{abstract}

\section{Introduction}

Let us introduce the following denotations: $B^{k}=\left(\nu^{k 1}, \ldots, \nu^{k n}\right)$, $\nu^{k i} \in[0,1], k, i \in I=\{1 \ldots n\} \quad$ is a preservation matrix of the $k$-th branch, $\quad C=\left(C^{i j}\right)_{i, j=1}^{n}, C^{i j}>0, i, j \in I=\{1 \ldots n\} \quad$ is a cost matrix coefficients, where $C^{i j}$ is a number of product of the $i$-th branch, necessary for the production of the unit product of the $j$-th branch. 
$F^{i}=\min \frac{x^{i j}}{C^{j i}}, \quad i, j \in I=\{1 \ldots n\}$ is a productive function of the $i$-th branch, $\ell=\left(\ell_{1}, \ldots, \ell_{2}\right)$ - price vector, $\mu^{k}-$ maximal growth rate of the total wealth of the $k$-th branch.

Then we can define the model $Z$ by the productive [1-4]:

$$
\begin{gathered}
a(X)=\left\{\tilde{X}=\left(\tilde{x}^{1}, \ldots, \tilde{x}^{n \cdot}\right) \in\left(R_{+}^{n}\right)^{n} \mid \sum_{k \in I}^{n} \tilde{x}^{k i} \leq \sum_{k \in I} \nu^{k i} \cdot x^{k i}+\min _{j \in I} \frac{x^{i j}}{c^{j i}},\right. \\
\left.x^{k \cdot}=\left(x^{k 1}, \ldots, x^{k n}\right), k \in I, \nu^{k i} \in[0,1], c^{i j}>0(i, j \in I)\right\} .
\end{gathered}
$$

Here we describe the construction of the trajectory of this model by the help of equilibrium mechanism without loss [4].

The working of the economy as a dynamic system, is given by the state $y$ at time $t$.

Note that equilibrium is a set $\left(P, x^{1}, \ldots, x^{n \cdot}, y\right)$, and if the equilibrium exists then the vector $x^{k}$ is definitely proportional to $c^{\cdot k}(k \in I)$. If $y \notin$ cone $\left\{c^{\cdot i} \mid i \in I\right.$, the equilibrium is obviously does not exist.

(1) If $y \in$ cone $\left\{c^{\cdot i} \mid i \in I\right\}$, then expanding it on the basis, we obtain $\sum_{i \in I} \gamma^{i} \cdot c^{i}$, moreover the vector type of $\alpha^{i} \cdot c^{i}(i \in I)$ are solutions of the consumer problem.

(2) If one may choose $\ell^{i}, \mu^{j}(i, j \in I)$ by the way that the following inequality would be satisfied:

$$
\ell^{i} \cdot v^{j i}+\frac{1}{|C|} \cdot \sum_{k \in I}(-1)^{i+k+1} \cdot \frac{\mu^{j}}{\mu^{k}}\left(\ell^{k}+\sum_{m \in I} \ell^{m} \cdot \nu^{k m} \cdot c^{m k}\right)\left|C_{i}^{k}\right| \leq 0,
$$


where $C_{i}^{k}$ is $(n-1) \times(n-1)$ matrix obtained from the matrix $C$ by removing the $k$-th column and $i$-th row, then equilibrium without loss exists.

Thereafter, according to $\bar{x}^{i \cdot}(i \in I)$ via preservation matrix $B^{i}$ and the production function $F^{i}$ yields the following vector of distributed resources $\tilde{y}$ at time $t+1$, and then the process is repeated.

\section{Main Part}

In [7], necessary and sufficient conditions are obtained for the existence of an equilibrium state without loss (see Theorems 3 and 4). But verification of necessary and sufficient conditions for the existence of equilibrium prices without loss is complicated enough - a system of $n^{2}$ linear inequalities (24) or system of $n$ superlinear inequalities (29) [7]. Therefore, it is advisable to derive the necessary conditions for the existence of equilibrium prices without loss.

Consider the special case when all $\mu^{j}(j \in I)$ are the same, that is, the branches grow with the same maximal rate of the total wealth.

Equilibrium prices without loss $P$ are solution of (12) [7]. By Theorem Fan Ji [8], the necessary conditions of absence of equilibrium prices without loss can be formulated as the following problem:

By which $\ell^{i}, \nu^{j i}, c^{i j}(i, j \in I)$ there exist $\nu^{j i} \geq 0$ and $u^{j}(i, j \in I)$, satisfying the inequality

$$
\sum_{j \in I} \nu^{j i}=-\sum_{j \in I} u^{j} \cdot c^{i j}, \quad \forall i \in I,
$$

for which is valid

$$
\sum_{j \in I}\left(\sum_{i \in I} \nu^{j i} \cdot \ell^{i} \cdot \nu^{j i}+u^{j} \cdot\left(\ell^{j}+\sum_{i \in I} \ell^{i} \cdot \nu^{j i} \cdot c^{i j}\right)\right)>0 .
$$


Let the prices $\ell=\left(\ell^{1}, \ldots, \ell^{n}\right)$ are normed by the relation

$$
\sum_{i \in I} \ell^{i}=1, \quad \ell^{i}>0(i \in I) .
$$

Consider the left hand side of (2) taking into account (3):

$$
\begin{gathered}
\sum_{j \in I}\left(\sum_{i \in I} \nu^{j i} \cdot \ell^{i} \cdot v^{j i}+u^{j} \cdot\left(\ell^{j}+\sum_{i \in I} \ell^{i} \cdot \nu^{j i} \cdot c^{i j}\right)\right) \\
=\sum_{j \in I}\left(\sum_{i \in I} \ell^{i} \cdot \nu^{j i} \cdot \nu^{j i}+u^{j} \cdot\left(\ell^{j} \sum_{i \in I} \ell^{i}+\sum_{i \in I} \ell^{i} \cdot \nu^{j i} \cdot c^{i j}\right)\right) \\
=\sum_{i \in I} \ell^{i} \cdot\left(\sum_{j \in I}\left[v^{j i} \cdot v^{j i}+u^{j} \cdot\left(\ell^{j}+v^{j i} \cdot c^{i j}\right)\right]\right) .
\end{gathered}
$$

Thus the inequality (2) takes the form

$$
\sum_{i \in I} \ell^{i} \cdot\left(\sum_{j \in I}\left[v^{j i} \cdot v^{j i}+u^{j} \cdot\left(\ell^{j}+v^{j i} \cdot c^{i j}\right)\right]\right)>0 .
$$

Let's introduce the set on indexes

$$
\begin{gathered}
J_{1}(i)=\left\{j \in I \mid \nu^{j i}=\max _{k \in I} \nu^{k i}\right\}, \quad \forall i \in I, \\
J_{2}(i)=\left\{j \in I \mid \ell^{j}+\nu^{j i} \cdot c^{i j}=\min _{k \in I}\left(\ell^{k}+\nu^{k i} \cdot c^{i k}\right)\right\}, \quad \forall i \in I .
\end{gathered}
$$

It takes place

Lemma 1. Let the conditions (3) and the inequality

$$
\frac{\max _{j \in I} \nu^{j i}}{\left|J_{1}(i)\right|} \cdot \sum_{j \in J_{1}(i)} c^{i j}>\min _{j \in I}\left(\ell^{j}+v^{j i} \cdot c^{i j}\right), \quad i \in I,
$$

are valid, where $\left|J_{1}(i)\right|$ is a number of elements in the set of indexes $J_{1}(i)$. In this case there exist the numbers $v^{j i} \geq 0$ and $u^{j}(i, j \in I)$, satisfying (1), for which (2) or (3) is fulfilled. 
Proof. Introduce the sets $J_{1}(i)$ and $J_{2}(i)$ by the formulas (5). Let the conditions (3) and (1) be satisfied. Then define $\nu^{j i}$ and $u^{j}(i, j \in I)$ as follows:

$$
\begin{gathered}
u^{j}=\left\{\begin{array}{l}
-\varepsilon^{i}, \text { if } j \in J_{2}(i), \quad(i \in I), \\
0, \text { if } j \notin J_{2}(i),
\end{array}\right. \\
\nu^{j i}=\left\{\begin{array}{c}
\frac{\varepsilon^{i}}{\left|J_{1}(i)\right|} \cdot \sum_{j \in J_{1}(i)} c^{i j}, \text { if } j \in J_{1}(i), \quad(i \in I), \\
0, \quad \text { if } j \notin J_{1}(i),
\end{array}\right.
\end{gathered}
$$

where $\varepsilon^{i}>0$ is some number $(i \in I)$.

Substituting the values $u^{j}$ and $v^{j i}(i, j \in I)$ into the condition (1), it is easy to see that it is satisfied.

Multiplying both sides of the inequality (6) by $\ell^{i} \cdot \varepsilon^{i}\left(\varepsilon^{i}>0\right)$ and summing over $i \in I$, we get

$$
\sum_{i \in I} \ell^{i} \cdot \varepsilon^{i} \cdot \max _{j \in I} \nu^{j i} \cdot \frac{1}{J_{1}(i)} \cdot \sum_{j \in J_{1}(i)} c^{i j}>\sum_{i \in I} \ell^{i} \cdot \varepsilon^{i} \cdot \min _{j \in I}\left(\ell^{j}+v^{j i} \cdot c^{i j}\right)
$$

From the other hand substituting the values $u^{j}$ and $v^{j i}(i, j \in I)$ into (4) (or (2)), considering (3), we get (7).

Lemma 1 is proved.

Theorem 1. Let the numbers $\nu^{j i} \geq 0, \ell^{i}>0(i, j \in I)$ be such that $\max _{j \in I} \nu^{j i}>0, \sum_{i \in I} \ell^{i}=1$ and $\mu^{j}=1(j \in I)$. If equilibrium prices without loss by given $v^{j i}, \ell^{i}$ and some $c^{i j}>0(i, j \in I)$ exist, then the following type inequality turns true: 


$$
\min _{i \in I}\left(\frac{\max _{j \in I} v^{j i}}{\left|J_{1}(i)\right|} \cdot \sum_{j \in J_{1}(i)} c^{i j}-\min _{j \in I}\left(\ell^{j}+v^{j i} \cdot c^{i j}\right)\right) \leq 0 .
$$

The proof immediately follows from Fan Ji [8] and Lemma 1.

Consider the Neumann-Gale model, given by production mapping $a$ :

$$
\begin{aligned}
a(X)= & \left\{\tilde{X}=\left(\tilde{x}^{1 \cdot}, \ldots, \tilde{x}^{n \cdot}\right) \in\left(R_{+}^{n}\right)^{n} \mid 0 \leq \sum_{k \in I} \tilde{x}^{i \cdot} \leq \sum_{k \in I} B^{k} \cdot x^{k}\right. \\
& \left.+\left(F^{1}\left(x^{1 \cdot}\right), \ldots, F^{n}\left(x^{n \cdot}\right)\right), x^{k \cdot}=\left(x^{k 1}, \ldots, x^{k n}\right), k \in I \equiv\{1,2, \ldots, n\}\right\},
\end{aligned}
$$

where $B^{k}$ is a diagonal matrix the main diagonal of which has a form

$$
\begin{aligned}
& \left(\nu^{k 1}, \ldots, \nu^{k n}\right), \quad \nu^{k i} \in[0,1], \quad k, i \in I ; \\
& F^{i}\left(x^{i \cdot}\right)=\min _{j \in I} \frac{x^{i j}}{c^{j i}}, \quad c^{j i}>0, \quad i, j \in I .
\end{aligned}
$$

We define the Neumann equilibrium state in this model $Z[1,5,6]$. Recall that the set $(\alpha, \bar{X}, \bar{P})$ is a Neumann equilibrium state, if the following conditions are satisfied:

$$
\begin{gathered}
\alpha \cdot \bar{X} \in a(\bar{X}), \\
{[\bar{P}, Y] \leq \alpha \cdot[\bar{P}, X] \quad(Y \in a(X)),} \\
{[\bar{P}, \bar{X}]>0,}
\end{gathered}
$$

where $a$ is a production mapping of the considered model $Z$.

Note that the prices of the products do not depend on the branches where they are considered, i.e., $\bar{P}=(P, \ldots, P)$, where $P=\left(P^{1}, \ldots, P^{n}\right)$.

Note that nondegenerating case means that 


$$
\alpha \cdot \sum_{k \in I} \bar{x}^{k i}=\sum_{k \in I} \nu^{k i} \cdot \bar{x}^{k i}+\min _{j \in I} \frac{\bar{x}^{i j}}{c^{j i}}, \quad \forall i \in I .
$$

Due to the fact that in the system (11) only strict equalities should be satisfied, therefore, we are dealing with equilibrium without a loss, i.e.,

$$
\frac{\bar{x}^{k 1}}{c^{1 k}}=\cdots=\frac{\bar{x}^{k n}}{c^{n k}}, \quad \forall k \in I .
$$

It follows from this

$$
\bar{x}^{k \cdot}=\sigma^{k} \cdot c^{\cdot k}, \quad k \in I .
$$

Assume that

$$
\ell=\frac{1}{\alpha} \cdot P, \quad \mu^{k}=1
$$

Note that $\mu^{k}$ is a maximal growth rate of the total wealth of $k$-th branch $[4,9,10]:$

$$
\mu^{k}=\max _{x \geq 0} \frac{U^{k}(\ell, x)}{[P, x]}, \quad k \in I
$$

where

$$
U^{k}\left(\ell, x^{k \cdot}\right)=\sum_{j \in I} \ell^{i} \cdot \nu^{k i} \cdot x^{k i}+\ell^{k} \cdot \min _{j \in I} \frac{x^{k i}}{c^{i k}}, \quad k \in I .
$$

Due (11) and (13) we have

$$
\alpha=\frac{U^{1}\left(P, \bar{x}^{1 \cdot}\right)}{\left[P, \bar{x}^{1 \cdot}\right]}=\cdots=\frac{U^{n}\left(P, \bar{x}^{n \cdot}\right)}{\left[P, \bar{x}^{n \cdot}\right]},
$$

where $\bar{X}=\left(\bar{x}^{1 \cdot}, \ldots, \bar{x}^{n \cdot}\right), \bar{P}=(P, \ldots, P)$.

The system of relations (14) one may obtain also from the condition (9) taking $X=\bar{X}$. 
Neumann equilibrium state $(\alpha, \bar{X}, \bar{P})$ is determined up to a factor, as the multiplication of any of the relations (8)-(10) by a positive factor does not change these relations (recall that $\alpha$ is a superlinear mapping). Therefore it is suitable to take $P^{1}=1, \sigma^{1}=1$ in the formula (12). From (12) follows, Neumann equilibrium vector $\bar{X}$ is defined by the parameters $\sigma^{1}, \ldots, \sigma^{n}$. Due to above considerations, we get that Neumann equilibrium state $(\alpha, \bar{X}, \bar{P})$ is defined by $2 n-1$ variablesç işeş by $\alpha, P^{2}, \ldots P^{n}, \sigma^{2}, \ldots, \sigma^{n}$. Note that

$$
\min _{j \in I} \frac{\bar{x}^{i j}}{c^{j i}}=\sigma^{i}, \quad i \in I .
$$

Then substituting (12) into (11) and (14), we obtain a system of $2 n-1$ equations with respect to $\alpha, P^{2}, \ldots, P^{n}, \sigma^{2}, \ldots, \sigma^{n}$ :

$$
\left\{\begin{array}{c}
\sum_{k \in I} \sigma^{k} \cdot c^{i k}=\sum_{k \in I} \sigma^{k} \cdot v^{k i} \cdot c^{i k}+\sigma^{i}, \quad(i \in I) \\
\frac{P^{1} \cdot \sigma^{1}+\sum_{i \in I} P^{i} \cdot \sigma^{i} \cdot \nu^{1 i} \cdot c^{i 1}}{\sigma^{1} \cdot \sum_{i \in I} P^{i} \cdot c^{i 1}}=\cdots=\frac{P^{n} \cdot \sigma^{n}+\sum_{i \in I} P^{i} \cdot \sigma^{i} \cdot \nu^{n i} \cdot c^{i n}}{\sigma^{n} \cdot \sum_{i \in I} P^{i} \cdot c^{i n}}=\alpha .
\end{array}\right.
$$

In addition to this system these variables should satisfy the inequality (10), which due to (12) takes the form

$$
\sum_{j \in I} P^{j} \cdot \sum_{i \in I} \sigma^{i} \cdot c^{j i}>0 .
$$

Thus in fact the following theorem is proved.

Theorem 2. Neumann equilibrium state that satisfies the Equation (11) can be constructed using the equilibrium model without loss by $\ell=\frac{1}{\alpha} \cdot P, \mu^{k}=1(k \in I)$, wherein the equilibrium can be found from the system (15) and (16). 
Note: Let Neumann prices $P=\left(P^{1}, \ldots, P^{n}\right)$ are normed by the condition $\left[P, \bar{x}^{k \cdot}\right]=1(k \in I)$.

In this case,

$$
\sigma^{k}=\frac{1}{\left[P, c^{\cdot k}\right]}, \quad k \in I
$$

Substituting (17) into (15) we obtain a system with respect to $n+1$ variables $\alpha, P^{1}, \ldots, P^{n}$.

\section{References}

[1] V. L. Makarov and A. M. Rubinov, Mathematical Theory of the Economical Dynamics and Equilibrium, Moscow, Nauka, 1973, 336 p.

[2] A. M. Rubinov, Mathematical Models of the Expanded Reproduction Models, Leningrad, Nauka, 1983.

[3] A. M. Rubinov, Superlinear Multivalued Mappinqs and their Applications to Economical-Mathematical Problems, Leningrad, Nauka, 1980.

[4] A. M. Rubinov, Equlibrium Mechanisms for Effective and Development of Dynamic Models of Production and Exchange, Technical Cybernetics, 1968.

[5] G. B. Kleiner, Production Functions, Moskow, 1986.

[6] I. A. Krass, The Models of Economic Dynamics, Moskow, 1976.

[7] S. I. Hamidov, On a consumer problem, Pure and Applied Mathematics Journal 5(6) (2016), 205-210.

[8] Fan Ji, On the Systems of Linear Inequalities, In the book 'Linear Inequalities and Close Problems”, Moscow, IL, 1969.

[9] R. M. Romer, Mathiness in the theory of economic growth, The American Economic Review 105(5) (2015).

[10] V. F. Demyanov and L. V. Vasilyev, Non-differentiable Optimization, Moskow, Nauka, 1981. 